\title{
Designing a Telecare Product for the Elderly
}

\author{
Obinna Onyimadu, Fraser Harding \\ Passivsystems Ltd \\ Newbury, UK \\ Obinna.Onyimadu@passivsystems.com
}

\author{
Dr Jim Briggs \\ Centre for Health Care Modelling and Informatics \\ University of Portsmouth \\ Portsmouth, UK \\ Jim.Briggs@ port.ac.uk
}

\begin{abstract}
In this paper, we describe our experience of designing a telecare product for the elderly. This is a collaborative project involving Passivsystems Ltd and the Centre for Healthcare Modelling and Informatics at the University of Portsmouth. The research aims at adapting Passivsystems' existing in-home hub so that it is capable of providing a telecare service in addition to its energy management service. Our work focuses on designing a user centred product around the recipient's Activities of Daily Living (ADL). We describe our research over 10 months comprising a description of our product model and the motivations behind the adopted model.
\end{abstract}

Keywords: Telecare, Activities of Daily Living (ADL), Activity Index, Knowledge Discovery

\section{INTRODUCTION}

The Business $+{ }^{1}$ project between the University of Portsmouth's Centre for Health Care Modelling and Informatics

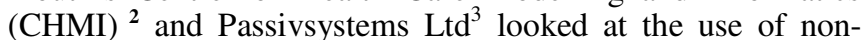
intrusive sensors in the design and development of the next generation assisted care services for elderly individuals. We are fast becoming an aging society[1] and the care and wellbeing of elderly people is a primary concern not just for the purpose of maintaining their independence but also for the purpose of reassuring family members and cost savings. Assisted care services would provide care professionals and loved ones with monitoring tools and means of evaluating the wellbeing of the individual. Primarily this service would provide reassurance to family members and empower carers with the ability to make better decisions and interventions.

Passivsystems' existing home energy management system consists of a hub and several sensors, including pipe and tank sensors connected to a Wired Sensor Communicator (WSC) that then communicates wirelessly with the hub via ZigBee. ZigBee temperature sensors monitor internal temperature. Data from these sensors (installed on the boiler, inflow and outflow pipes) are prepared and mined to manage the individual's home heating and hot water usage. Part of the system's capability is the ability to intelligently fire the home owner's boiler to a set point by learning the home thermal properties and hot water usage. Having such a system in a home provides a clear avenue for deploying an assisted living platform given the common

Business+ is a South East England Development Agency (SEEDA) funded scheme designed to improve the competitiveness of business through the use of knowledge and research from universities within the UK.

http://www.chmi.port.ac.uk

http://www.passivsystems.com technological base shared by both telecare services and smart home systems [2].

Telecare delivery models can be Reactive or Proactive. Reactive systems respond to events in the home. They provide reports and notifications and have no inherent intelligence. The hardware used in this sort of set up commonly consists of sensors that raise alerts when triggered. Sensors within this category are environmental sensors such as temperature sensors, flood sensors, smoke detectors, etc. This sort of system is flexible and takes into account factors such as home architecture, ergonomics and personal preference. It also fits well into the whole concept of delivering care based on the results of the recipient's care assessment (Telecare delivery requires a careful assessment of the care recipient's needs [3]. Eligibility criteria ${ }^{4}$ enable care practitioners to properly assess the needs of the care recipient). It is thus easy to assemble hardware for the purpose of monitoring various risk conditions so that for two people with similar conditions the choice of hardware and installation could vary. Curry et al. [4] describe this situation with a simple phrase: "One size does not fit all". Proactive systems extend reactive systems. They offer more intelligence by monitoring trends and looking for patterns in the recipient's lifestyle so that the carer or family member can make an appropriate and timely intervention. Knowledge discovery techniques can be employed in such a system, for example to analyse the frequency of the bed sensor firing as a means of measuring the quality of sleep [5] or activity recognition by observing water usage in the home [6].

In this paper we suggest a new approach that is more proactive and extends the eligibility criteria by designing and deploying telecare around the recipient's care plan. A care plan is an agreement between an individual and a health professional to help manage a person's day-to-day health. This way we not only monitor and report events, we also monitor set goals, e.g. exercising, performing Activities of Daily Living (ADLs), etc. in such a way that the wellbeing of the individual improves over a period of time.

\section{PREVIOUS WORK}

Telecare solutions have evolved from first and second generation systems (systems designed to alert a carer of an event) to third generation systems. Third generation systems are called preventive systems and were born out of advances in sensor technology and a continuing reduction in the cost of processing data[7]. Research on third generation systems has focused on

\footnotetext{
${ }^{4}$ A set of guidelines used by local councils in assessing the level of care re-
} quired by an individual. 
monitoring wellbeing and everyday ADLs. However ADL monitoring can be very complex[8] as human behaviour is both unpredictable and people can do more than one thing at the same time. In spite of this, research on automatic activity detection has produced very interesting results from using machine learning algorithms and an array of sensors. For instance, in the MIT PlaceLab project[9], ADLs such as bathing, toileting and grooming were detected with accuracies of between $25 \%-89 \%$ depending on the evaluation criteria used[10]. Since the test was carried out in multiple residences over 70 sensors were deployed in each house.

The difficulty in inferring or recognizing all the ADLs, supplemented by the fact that some ADLs have a stronger relationship to well-being[11], has led to the research and development of activity recognition algorithms for specific ADLs. For instance, Wan [12] used Radio-Frequency-Identification (RFID) as contact switches to infer when users took medication. Phillipose [13] suggests that these systems have limited inferencing and lack synergy and interconnection, saying that "research tuned to detecting hand washing or tooth brushing has had nearly no synergy, each using its own idiosyncratic sensors and algorithms". The (Independent Lifestyle Assistant) ILSA Project was designed to counter this lack of synergy by monitoring a wider range of ADLs using an agent based system[14].

One of the challenges with activity recognition algorithms is having multiple residents in the same environment simultaneously[9, 15]. Another practical challenge lies in the sheer number of sensors required to gather enough quality data capable of developing a good activity model. For instance, in the MIT PlaceLab[10], over 70 sensors are deployed on doors, home utensils, machines, etc. The practicality of this approach in real life terms isn't feasible. Important factors such as the ergonomics of the home and the stigma attached[16] to the occupant of a home with such a large number of sensors is a subject of concern. These issues are perhaps the reason for the slow adoption of ADL detection in commercial applications.

\section{METHODOLOGY}

Our research methodology was guided by our knowledge of the product recipients: elders, carers and family members. We identified issues affecting the independence of elderly people as well as issues of concern to their carers and family members. This would guide us in our design and choice of sensor technologies. According to Curry[4], two categories of elders exist: the Autonomous Individual and the Vulnerable Individual. Within the autonomous category are those "with mild and moderate symptoms and those who are currently healthy but have a predisposition to Long Term Conditions (LTCs)". The vulnerable individual on the other hand is different in that "the subject to be monitored is deemed to be vulnerable or incapable in some way, either through the general frailties of old age or because of early onset of dementia"[4]. The autonomous individual still has good cognitive senses and is in a good enough condition to live independently, however family members do not want to be caught unawares when the elder's cognitive or physical abilities begin to fail. We discover then that there exists a wide spectrum of concerns and risks of which elders are susceptible, spanning time and varying in use case. We re- viewed existing papers and white papers to come up with a matrix of common risks and associated conditions.

Ideally, the next stage would be to begin the design of the system. However, while this approach is commonly adopted by researchers and commercial products, we discovered certain inherent flaws. Firstly because of the different conditions and varying risks, deciding what the product would monitor or what risk factors it would cater for becomes difficult. The natural thing would be to design a sort of general system that looks after basic conditions and risk factors, and then provide extra capabilities which meet unique needs. However determining what constitutes a general system is subjective. Secondly, this approach is liable to limit the eventual product to a particular condition. The reasoning behind this is that if the product cannot be designed to address all the risks and conditions, it could be designed to address only one sort of risk. In addition, the risk of stigmatisation arises when designing the product for a particular condition.

\section{A. Care Plan}

In light of the issues highlighted in the previous section, we sought a new approach which was to design the product around the elder's care plan. It can be a written document or something recorded in a patient's notes[17]. At the point of care delivery, a care plan is produced by the GP or occupational therapist for the elder based on an assessment of their health and wellbeing.

This care plan consists of a regimen of activities, do's and don'ts with clearly stipulated objectives and goals set by both the individual and the Occupational Therapist (OT), doctor, carer, etc. By monitoring the individual's compliance with the objectives of the care plan, this approach would provide carers (family and friends) and the individual with a more objective and focused assessment of their health and day to day life. The difference between this approach and the conventional one (where care is based on the individual's condition) lies more in the objective and aims. While the same sensor technologies will be used in both approaches, the objective of our approach is to check for compliance using various assessment measuring schemes and then to enable carers to tell the individuals which areas they have to improve on in order to maintain a certain state of health.

For instance, consider this case study: Mrs Smith is 70 years old and has Parkinson's disease. Her doctor and OT have formulated her care plan, with some input from her family. The excerpt below is adapted from [18]:

\section{Name: Mrs Jane Smith \\ Date of Evaluation: 31/03/2010 \\ Area: Fitness and well being}

Current Situation: Jane was recently discharged from the hospital. She looks frail from some weight loss. She also has problems sleeping at night.

Objective: To ensure that Jane receives optimum nutrition to maintain stable weight, e.g. maintain $55 \mathrm{~kg}$ for two weeks.

Action: Ensure Jane receives a healthy and regular diet. Go for regular walks to exercise her muscles. Ensure Jane goes to bed thirty minutes after dinner. 
Mrs Smith's care plan shows her current situation, her objective, and the actions required to meet the objectives. In our approach, we use sensors to monitor her such that feedback sent to the carer and family informs them if she is doing the actions that meet her objectives. We would use assessment scales to provide a numerical (and possibly graphical) measure of how well the individual is complying with the regime. The usefulness of this approach extends to the fact that the care plan should cover every area of the individual's wellbeing because they are interconnected. For instance a decline in sleep patterns will influence cognitive abilities and consequently activities of daily living. We see then that by observing deviations from the care plan in a particular area, the carer might be able to predict subsequent deviations in other areas and then take immediate action. In addition, it deals with the problem of noncompliance with the prescribed care. Noncompliance in healthcare is a significant problem. The rate of compliance with medication is about 50\%[19], and much lower for lifestyle predictions and other behavioural demanding regimens. Deviations in the regime can be detected easily and responded to by the carer or family member through an interface or reporting tool.

\section{B. Care Assessment Scales}

Since our approach relies on assessing the elder's development in line with meeting the care objectives, measurable indicators are required to give a clear indication of their state. We want a numerical score which gives a measure of whatever is assessed. A number of assessment models exist (see Table 1) and they assess a range of wellbeing constituents such as morale, ability to perform activities of daily living, memory, etc. Our design aims at monitoring the wellbeing of the individual, so we make use of the ADL scale because it covers most aspects of the individual's lifestyle.

TABLE I. RECOMMENDED ASSESSMENT SCALES FOR THE ELDERLY

\begin{tabular}{|l|l|}
\hline \multicolumn{1}{|c|}{ Function } & \multicolumn{1}{c|}{ Assessment Scale } \\
\hline Primary ADL & Berthel ADL Index[20], Katz ADL, Lawton IADL \\
\hline $\begin{array}{l}\text { Memory and } \\
\text { Cognition }\end{array}$ & Abbreviated Mental Test [21], FIM + FAM \\
\hline Communication & Lambeth Questionnaire Screening Questions [22] \\
\hline Morale & Philadelphia Geriatric Center Morale Scale[23] \\
\hline Social Status & Social Status Checklist \\
\hline Mood & Geriatric Depression Scale [24] \\
\hline
\end{tabular}

\section{Wellbeing Assessment}

In our design, we create an Activity Index (AI) which is a measure of the elder's wellbeing on a scale of 1 to 100 . The AI is derived from the ADL and is a sum total of a number of factors such as:

- Motion/mobility/immobility: How much movement or lack of movement is detected in the home. We intend to model the person's activities by obtaining daily routine from carers and later using learning algorithms. Deviations in this activity level would influence the AI.
- Bathroom Activity.

- Medication: Taking medication at the wrong time or taking incorrect dosage might influence the wellbeing of the individual, so detecting that the individual has taken or not taken his medication and incorporating that in the $\mathrm{AI}$ is quite important.

- $\quad$ Sleep.

Currently we are working on algorithms capable of calculating the AI.

\section{System Design}

Our final product will feature a number of sensors and actuators in the individual's home. Since our design hinges on wellbeing and compliance, the sensors and actuators will be configured to monitor and report on:

- Activity: Motion (e.g. PIR) sensors will be used in detecting activity and inactivity. Data from these would be analysed to compute the AI. Based on the care plan, carers can pre-configure the system to report on particular activities. For instance, the elder might be required to take a walk every evening at approximately $6 \mathrm{pm}$. A door sensor at the entrance of the home will report entry and exit times. If there is no exit by $7 \mathrm{pm}$, the system can be preconfigured to report this event to the carer or family member.

- Medication intake: Compliance to medication is a significant part of telecare. The elder might be at risk of taking the wrong medication, forgetting to take the medication completely, or failing to take the medication at the scheduled time. Using medication dispensers and reminders, medication intake can be managed and its effect on the elder can also be monitored from data collected.

- Night time sleep: The elder's quality of sleep will greatly influence his/her wellbeing. We can measure it by the duration of the sleep, when the elder went to bed and when he/she woke up, the number of sleep interruptions, e.g. how often the elder left the bed, and even the frequency of twists and turns. Attaching a bed sensor would tell us when the elder went to bed and show the number of interruptions. In addition, twists and turns would also be identified through the bed sensor or a worn device like the wrist-care watch[6]. By analysing the data from these devices, we can develop a model for sleep pattern, predicting the quality of sleep. We can also observe trends in sleep duration and wake up time by plotting and comparing daily, weekly and monthly data. This sort of information would enable carers to observe if the elder is meeting the requirements of the care plan relating to sleep and also make timely intervention.

- Falls: Falls could be recovered or unrecovered. Our system would make use of data from a combination of motion sensors, fall sensors or pendants with inbuilt accelerometers to differentiate and report falls. The time of the fall and the duration for which the elder 
remained on the floor would be logged in the system and reported to the carer or family member.

- Kitchen events: Kitchen activity would ideally suggest food preparation. Contact sensors could be installed on the fridge or microwave. Motion sensors installed in the kitchen would detect activity. Water inflow and outflow in the kitchen sink can also be monitored. Data from these sensors would be fed into a model to determine if the elder is cooking or not. The elder's care plan might stipulate meal times. Sensor events could be analysed to determine the elder's compliance to the diet regimen.

- Events: Unusual occurrences or events are capable of exposing the elder to danger; for instance, the front door opening at odd hours or a flood in the bathroom or kitchen. Environmental sensors such as for flood, carbon monoxide and smoke can be set up to alert of these dangers. Monitoring these sorts of events can inform the carer or family member of the elder's cognitive abilities - e.g. frequently forgetting to turn off the tap resulting in flooding could suggest that the elder needs more care.

\section{NEXT STEPS}

Future work involves development and trials. We have already commenced this by installing several PIRs in a test home and analysing the data from these sensors. The test individual records all his activities. Our objective is to develop algorithms and models for determining activity index. Already, ethical issues have arisen such as who can view the test subject's data? What happens to the data collected if the elder decides to leave the system? How much information is revealed and to who? To address these we will design our model around the important principles of dignity and respect, determining the exact bounds of these in discussions with representative users (elders and their carers).

\section{CONCLUSION}

In this paper, we have suggested a unique approach to delivering a proactive telecare system that monitors trends in the elder's lifestyle and proactively checks the daily wellbeing of the elder. Unlike other approaches which focus on delivering care for particular conditions or for monitoring purposes, our design focuses on maintaining and improving wellbeing through an implementation of the elder's care plan objectives providing the carer with ratings and scores that give a true impression of the elder's wellbeing. Our design also tackles the problem of compliance in care delivery.

\section{REFERENCES}

[1] M. A. Blythe, A. F. Monk, and K. Doughty, "Socially dependable design: The challenge of ageing populations for HCI," Interacting with Computers, vol. 17, pp. 672-689, Dec 2005.

[2] P. Tang and T. Venables, "'Smart' homes and telecare for independent living," Journal of Telemedicine and Telecare, vol. 6, pp. 8-14, 2000.

[3] B. G. Celler, W. Earnshaw, E. D. Ilsar, L. Betbedermatibet, M. F. Harris, R. Clark, T. Hesketh, and N. H. Lovell, "Remote Monitoring of HealthStatus of the Elderly at Home - A Multidisciplinary Project on Aging at the University of New-South-Wales," International Journal of BioMedical Computing, vol. 40, pp. 147-155, 1995.

[4] R. G. Curry, K. Lethbridge, and D. R. Parry, "An International Centre of Excellence in Telecare - ICE-T Background and Development," 2009.

[5] IST International Security Technology Oy, "WristCare Activity Data and its Practical Uses," 2005 http://www.obsmedical.com/wpcontent/uploads/2010/03/Application-guide-activity-curve-v1.2.pdf.

[6] J. Fogarty, C. Au, and S. E. Hudson, "Sensing from the basement: a feasibility study of unobtrusive and low-cost home activity recognition," in Proceedings of the 19th annual ACM symposium on User interface software and technology Montreux, Switzerland: ACM, 2006.

[7] B. A. Majeed and S. J. Brown, "Developing a well-being monitoring system-Modeling and data analysis techniques," Appl. Soft Comput., vol. 6, pp. 384-393, 2006.

[8] U. Naeem and J. Bigham, "A Comparison of Two Hidden Markov Approaches to Task Identification in the Home Environment," in Pervasive Computing and Applications, 2007. ICPCA 2007. 2nd International Conference on, 2007, pp. 383-388.

[9] B. Logan, J. Healey, M. Philipose, E. M. Tapia, and S. Intille, "A longterm evaluation of sensing modalities for activity recognition," UbiComp 2007: Ubiquitous Computing, Proceedings, vol. 4717, pp. 483-500, 2007.

[10] E. M. Tapia, S. S. Intille, and K. Larson, "Activity Recognition in the Home Using Simple and Ubiquitous Sensors," in Pervasive Computing. vol. 3001, A. Ferscha and F. Mattern, Eds.: Springer Berlin / Heidelberg, 2004, pp. 158-175-175.

[11] A. Sixsmith, N. Hine, I. Neild, N. Clarke, S. Brown, and P. Garner, "Monitoring the Well-being of Older People," Topics in Geriatric Rehabilitation, vol. 23, pp. 9-23, 2007.

[12] D. Wan, "Magic Medicine Cabinet: A Situated Portal for Consumer Healthcare," pp. 352-355.

[13] M. Philipose, K. P. Fishkin, M. Perkowitz, D. J. Patterson, D. Fox, H. Kautz, and D. Hahnel, "Inferring activities from interactions with objects," Ieee Pervasive Computing, vol. 3, pp. 50-57, 2004.

[14] K. Z. Haigh, L. M. Kiff, J. Myers, V. Guralnik, C. W. Geib, J. Phelps, and T. Wagner, "The independent lifestyle assistant\\&\8482; (I.L.S.A.): AI lessons learned," in Proceedings of the 16th conference on Innovative applications of artifical intelligence San Jose, California: AAAI Press, 2004, pp. 852-857.

[15] A. S. Crandall and D. J. Cook, "Coping with multiple residents in a smart environment," J. Ambient Intell. Smart Environ., vol. 1, pp. $323-$ 334, 2009.

[16] L. S. Mitteness and J. C. Barker, "Stigmatizing a Normal Condition Urinary-Incontience in Late-Life," Medical Anthropology Quarterly, vol. 9, pp. 188-210, 1995.

[17] NHS Choices, "What is a care plan?," http://www.nhs.uk/Planners/YourHealth/Pages/CarePlan.aspx Accessed 24th February 2011.

[18] Cornford House, "Care plan for Mrs Jane Smith."

[19] R. B. Haynes, "Improving patient adherence: state of the art, with a special focus on medication taking for cardiovascular disorders," in Patient compliance in healthcare and research, L. E. Burke and I. S. Okene, Eds. Armonk, NY: Futura Publishing, 2001, pp. 3-21.

[20] C. Collin, D. T. Wade, S. Davies, and V. Horne, "The Barthel ADL Index: a reliability study," Int Disabil Stud, vol. 10, pp. 61-3, 1988.

[21] H. M. Hodkinson, "Evaluation of a Mental Test Score for Assessment of Mental Impairment in the Elderly," Age and Ageing, vol. 1, pp. 233-238, 1972.

[22] H. Peach, S. Green, D. Locker, S. Darby, and D. L. Patrick, "Evaluation of a postal screening questionnaire to identify the physically disabled," Int Rehabil Med, vol. 2, pp. 189-93, 1980.

[23] M. P. Lawton, "Philadelphia Geriatric Center Morale Scale - Revision," Journals of Gerontology, vol. 30, pp. 85-89, 1975.

[24] J. A. Yesavage, T. L. Brink, T. L. Rose, O. Lum, V. Huang, M. Adey, and V. O. Leirer, "Development and Validation of a Geriatric Depression Screening Scale - A preliminary report," Journal of Psychiatric Research, vol. 17, pp. 37-49, 1983. 